\title{
Facilitating the Use of Epidemiological Models for Infectious Disease Surveillance
}

\author{
Alina Deshpande* and Kristen Margevicius \\ Analytics Intelligence and Technology, Los Alamos National Laboratory, Los Alamos, NM, USA
}

\section{Objective}

1. To develop a comprehensive model characterization framework to describe epidemiological models in an operational context.

2. To apply the framework to characterize "operational" models for specific infectious diseases and provide a web-based directory, the biosurveillance analytics resource directory (BARD) to the global infectious disease surveillance community.

\section{Introduction}

Epidemiological modeling for infectious disease is useful for disease management and routine implementation needs to be facilitated through better description of models in an operational context. A standardized model characterization process that allows selection or making manual comparisons of available models and their results is currently lacking. Los Alamos National Laboratory (LANL) has developed a comprehensive framework that can be used to characterize an infectious disease model in an operational context. We offer this framework and an associated database to stakeholders of the infectious disease modeling field as a tool for standardizing model description and facilitating the use of epidemiological models. Such a framework could help the understanding of diverse models by various stakeholders with different preconceptions, backgrounds, expertise, and needs, and can foster greater use of epidemiological models as tools in infectious disease surveillance.

\section{Methods}

We define, "operational" as the application of an epidemiological model to a real-world event for decision support and can be used by experts and non-experts alike. The term "model" covers three major types, risk mapping, disease dynamics and anomaly detection.

To develop a framework for characterizing epidemiological models we collected information via a three-step process: a literature search of model characteristics, a review of current operational infectious disease epidemiological models, and subject matter expert (SME) panel consultation. We limited selection of operational models to five infectious diseases: influenza, malaria, dengue, cholera and foot-and-mouth disease (FMD). These diseases capture a variety of transmission modes, represent high or potentially high epidemic or endemic burden, and are well represented in the literature. We also developed working criteria for what attributes can be used to comprehensively describe an operational model including a model's documentation, accessibility, and sustainability.

To apply the model characterization framework, we built the BARD, which is publicly available (http://brd.bsvgateway.org). A document was also developed to describe the usability requirements for the BARD; potential users (and non-users) and use cases are formally described to explain the scope of use.

\section{Results}

1. Framework for model characterization

The framework is divided into six major components (Figure 1): Model Purpose, Model Objective, Model Scope, Biosurveillance (BSV) goals, Conceptual Model and Model Utility; each of which has several sub-categories for characterizing each aspect of a model.

\section{Application to model characterization}

Models for five infectious diseases - cholera, malaria, influenza, FMD and dengue were characterized

using the framework and are included in the BARD database. Our framework characterized disparate models in a streamlined fashion. Model information could be binned into the same categories, allowing easy manual comparison and understanding of the models.

3. Development of the BARD

Our model characterization framework was implemented into an actionable tool which provides specific information about a model that has been systematically categorized. It allows manual categoryto-category comparison of multiple models for a single disease and while the tool does not rank models it provides model information in a format that allows a user to make a ranking or an assessment of the utility of the model.

\section{Conclusions}

With the model characterization framework we hope to encourage model developers to start describing the many features of their models using a common format. We illustrate the application of the framework through the development of the BARD which is a scientific and non-biased tool for selecting an appropriate epidemiological model for infectious disease surveillance. Epidemiological models are not necessarily being developed with decision makers in mind. This gap between model developers and decision makers needs to be narrowed before modeling becomes routinely implemented in decision making. The characterization framework and the tool developed (BARD) are a first step towards addressing this gap.

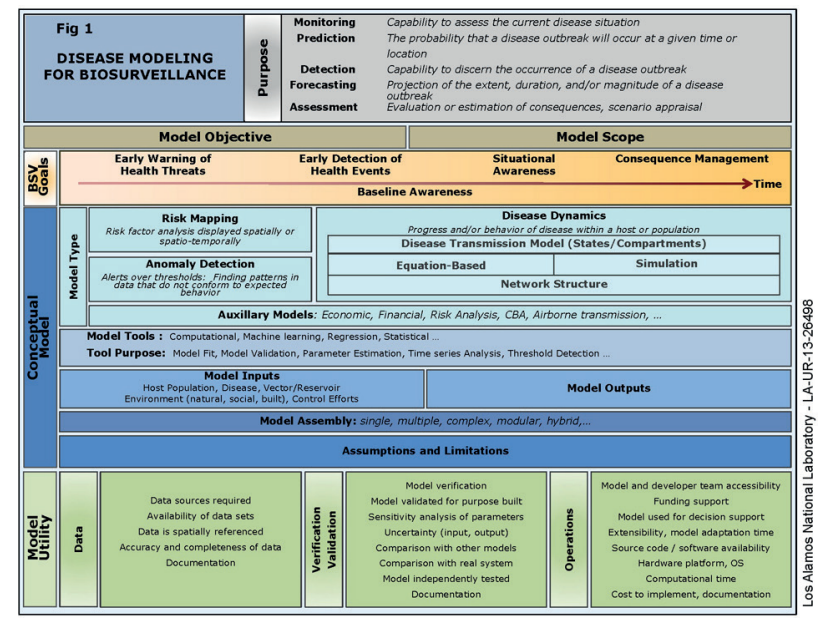

Keywords

epidemiological models; database; decision support 


\section{Acknowledgments}

Thanks to Dr. Madhav Marathe, Dr. Bryan Lewis, Dr. Steven Eubank (Virginia Bioinformatics Institute) and Dr. Reid Priedhorsky (LANL) for their advice and providing information for the characterization of the Epi-Simdemics model.

\section{References}

1. Hoshen MB, Morse AP. A weather-driven model of malaria transmission. Malaria J. 2004; 3: 32.

2. Kulldorrf M, Nagarwalla N. Spacial disease clusters: detection and inference. Stat Med. 1995; 14: 799-810. PMID: 7644860

3. Barrett CL, Bisset KR, Eubank SG, Feng X, Marathe MV. EpiSimdemics: an efficient algorithm for simulating the spread of infectious disease over large realistic social networks. International Conference for High Performance Computing, Networking, Storage and Analysis. 2008. SC 2008; Available: http://ieeexplore.ieee.org/ xpls/abs_all.jsp?arnumber $=5214892 \& \operatorname{tag}=1$.

\section{*Alina Deshpande}

E-mail: deshpande_a@lanl.gov 